\title{
BMJ Open Intrapartum and neonatal mortality among low-risk women in midwife-led versus obstetrician-led care in the Amsterdam region of the Netherlands: a propensity score matched study
}

\author{
Melanie M J Wiegerinck, ${ }^{1}$ Birgit Y van der Goes, ${ }^{2}$ Anita C J Ravelli, ${ }^{1,3}$ \\ Joris A M van der Post, ${ }^{1}$ Fayette C D Buist, ${ }^{4}$ Pieter Tamminga, ${ }^{5}$ Ben W Mol ${ }^{6,7}$
}

To cite: Wiegerinck MMJ, van der Goes BY, Ravelli ACJ, et al. Intrapartum and neonatal mortality among low-risk women in midwife-led versus obstetrician-led care in the Amsterdam region of the Netherlands: a propensity score matched study. BMJ Open 2018;8:e018845. doi:10.1136/ bmjopen-2017-018845

- Prepublication history and additional material for this paper are available online. To view these files, please visit the journal online ( $h$ ttp://dx.doi. org/10.1136/bmjopen-2017018845).

Received 29 July 2017 Revised 25 September 2017 Accepted 20 November 2017

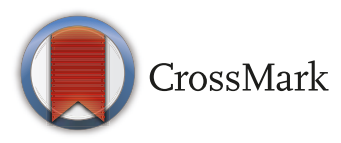

For numbered affiliations see end of article.

Correspondence to Melanie M J Wiegerinck; m.m.wiegerinck@amc.uva.nl

\section{ABSTRACT}

Objective To compare intrapartum and neonatal mortality in low-risk term women starting labour in midwife-led versus obstetrician-led care.

Study design We performed a propensity score matched study using data from our national perinatal register, completed with data from medical files. We studied women without major risk factors with singleton pregnancies who gave birth at term between 2005 and 2008 in the Amsterdam region of the Netherlands. Major risk factors comprised non-vertex position of the fetus, previous Caesarean birth, hypertension, (gestational) diabetes mellitus, post-term pregnancy ( $\geq 42$ weeks), prolonged rupture of membranes ( $>24$ hours), vaginal bleeding in the second half of pregnancy or induced labour. Groups were devided by midwife-led versus obstetrician-led care at the onset of labour. The primary outcome was intrapartum and neonatal ( $<28$ days) mortality. Secondary outcomes included obstetric interventions, 5 min Apgar scores $<7$ and neonatal intensive care admittance for $>24$ hours.

Results We studied 57396 women. Perinatal mortality occurred in 30 of $46764(0.64 \%$ ) women in midwife-led care and in 2 of $10632(0.19 \%$ ) women in obstetricianled care (OR $3.4,95 \% \mathrm{Cl} 0.82$ to 14.3 ). A propensity score matched analysis in a 1:1 ratio with 10632 women per group revealed an OR for perinatal mortality of $4.0(95 \% \mathrm{Cl}$ 0.85 to 18.9).

Conclusion Among low-risk women, midwife-led care at the onset of labour was associated with a statistically nonsignificant higher mortality rate.

\section{INTRODUCTION}

The Dutch obstetric system is characterised by a formal distinction between primary care (led by midwives or general practitioners) and secondary care (led by obstetricians). This distinction is based on an 'Indication' list in which conditions are defined for which women should be referred to different levels of care. ${ }^{1}$ Pregnant women without risk factors usually deliver in midwife-led care, although they can opt for obstetrician-led

\section{Strengths and limitations of this study}

- An important strength of our study is that we thoroughly checked for the completeness of our aggregated data with additional sources of the medical records.

- We created comparable groups through propensity score matching, thereby attempting to reduce bias due to confounding variables such as age, parity, socioeconomic status and ethnicity.

- Although our denominators were substantial, the large $\mathrm{Cls}$ in our primary mortality analyses overlapping 1 indicate limited power.

- Women were considered low risk if they had none of the predefined major risk factors, and because this was not always reliably registered in our national database we included a sensitivity analysis and further aimed to reduce imbalance in baseline characteristics between the groups with propensity score matched analysis (PSA).

- Our data refer to the period 1 January 2005 up to 31 December 2007, and although we know mortality rates have decreased since then, the 2010 ranking relative to other European countries showed only modest progression and need for further for improvement.

care. Women are referred when complications or risk factors occur either during pregnancy or labour. Women who start labour in midwife-led care can opt for home birth or hospital birth. Approximately half of all term women start labour in midwife-led care. ${ }^{2}$

Previous nationwide studies in the Netherlands indicated no differences in perinatal mortality among women who started labour in midwife-led care at home versus midwife-led care in hospital. ${ }^{34}$ Similar results were found in a Canadian study by Janssen et al $(0.4 \%$ vs $0.6 \%$; relative risk (RR) $0.61,95 \%$ CI 0.06 to $5.9),{ }^{5}$ with concomitant lower intervention 
rates (eg, Caesarean section (CS) rates in the intended home birth group of $7 \%$ vs $11 \%$; RR $0.76,95 \%$ CI 0.64 to $0.91)$, consistent with other studies. ${ }^{5-7}$

To our knowledge, the comparison of perinatal mortality in midwife-led (primary) versus obstetrician-led (secondary) care (with a clear description of both groups) was addressed in two Dutch studies. Evers $e t a l^{8}$ reported an increased intrapartum and early neonatal mortality risk in the midwife-led group (1.39\%o vs $0.60 \%$; unadjusted RR 2.3; $95 \%$ CI 1.1 to 4.8 ). This difference could not be confirmed in a similar study done by our group in the Amsterdam region $(0.70 \%$ vs $0.80 \%$; unadjusted RR $0.88 ; 95 \%$ CI 0.52 to 1.46$)$. However, both studies compared 'low-risk' women in midwife-led care to a combined population of 'low-risk' and 'high-risk' women in obstetrician-led care.

We therefore compared midwife-led care and obstetrician-led care in 'low-risk' women in the Amsterdam region, 'North-West Netherlands', where 19\% of all births in the Netherlands take place.

\section{MATERIALS AND METHODS}

Methods used to assemble our cohort have been described previously. ${ }^{9}$ In short, we performed a retrospective cohort study with use of linked data from the national perinatal register (PRN), together with additionally retrieved medical data from hospitals and midwifery practices. The PRN database is a national linked database in which $96 \%$ of the births in the Netherlands are registered. ${ }^{10}$ Data from the pregnancies with a general practitioner as the primary maternity caregiver (without referral to specialist care) were not registered in the database and therefore not included. ${ }^{11}$

We studied women with singleton pregnancies who gave birth beyond $37^{+0}$ weeks gestation. We excluded women with a preplanned CS, with fetal congenital anomalies or antepartum fetal death. We studied only women without any of the following predefined risk factors: non-vertex position of the fetus, previous Caesarean birth, hypertension, (gestational) diabetes mellitus, post-term pregnancy ( $\geq 42$ weeks), prolonged rupture of membranes ( $>24$ hours), vaginal bleeding in the second half of pregnancy or induced labour.

We selected women from the PRN database who gave birth at term between 1 January 2005 and 31 December 2007 in 'the perinatal region of Amsterdam'. This region contains 18 hospitals with obstetric/paediatric care facilities, which form Perinatal Cooperation Groups with their surrounding community practices of independent midwives and general practitioners. ${ }^{11}{ }^{12}$ Level of care comprised primary care (led by midwives or general practitioners, further referred to as midwife-led care) and secondary care (led by obstetricians; obstetrician-led care). Women can be referred to obstetrician-led care at any moment during pregnancy, birth or postpartum. In obstetrician-led care, deliveries can be done by obstetricians, but also by residents or so-called clinical midwives (always under responsibility of an obstetrician), usually with continuous cardiotocography (CTG) monitoring and immediate access to other medical facilities if needed. In this study, groups were formed by level of care at the onset of labour. In order to mimic an intention-to-treat approach, women who were transferred to obstetrician-led care during labour were analysed in the midwife-led care group.

\section{Identification of perinatal deaths}

First, we selected all women from the above-defined cohort whose babies suffered a perinatal death that was registered in the PRN. Subsequently, all 18 hospitals (obstetric and neonatal departments) in the region were requested to supply data regarding their perinatal deaths at term in the study period, which usually had been collected in their annual reports. We hand-searched all the annual reports to reduce the missing data. The retrieved supplementary data were added to the deaths that were identified from the PRN. In addition, cases classified in the PRN as antenatal stillbirth, congenital anomalies or multiple pregnancy, although not subject of this study, were verified for eligibility to double-check the classification from the PRN and the annual reports. Patient medical files were retrieved from the hospitals and midwifery practices, and were examined by an expert panel (midwife in primary care, midwife in secondary care, obstetrician and medical researcher) for detailed classification.

They were unaware of the classification in the PRN. Only cases of intrapartum or neonatal ( $<28$ days) death, without congenital anomalies, were considered for further selection, and cases with any of the previously mentioned predefined risk factors were then excluded. We anticipated that in some cases it would be impossible to distinguish antepartum and intrapartum death and defined an extra group of 'unable to classify' cases, to be addressed in a sensitivity analysis.

For our secondary outcomes, we collected PRN data regarding neonatal morbidity $(5 \mathrm{~min}$ Apgar scores below 7; admission to the neonatal intensive care (NICU) $\geq 24$ hours) and obstetrical interventions (mode of birth, use of epidural, perineal trauma, postpartum haemorrhage defined as reported blood loss $\geq 1000 \mathrm{~mL}$ and manual placental removal).

\section{Data analysis}

We used propensity score matched (PSM) pairs analysis to optimise the balance of baseline covariates between groups. ${ }^{13-15}$ Analyses were conducted separately for the unmatched (before PSM) and matched (after PSM) cohorts.

We generated the propensity scores of the probability of the smallest group (the obstetrician-led care group) at the onset of labour by logistic regression based on all available and relevant baseline covariates that were known from this pregnancy (listed in table 1). We used a one-to-one nearest neighbour matching without replacement. Propensity score distribution was plotted to check 


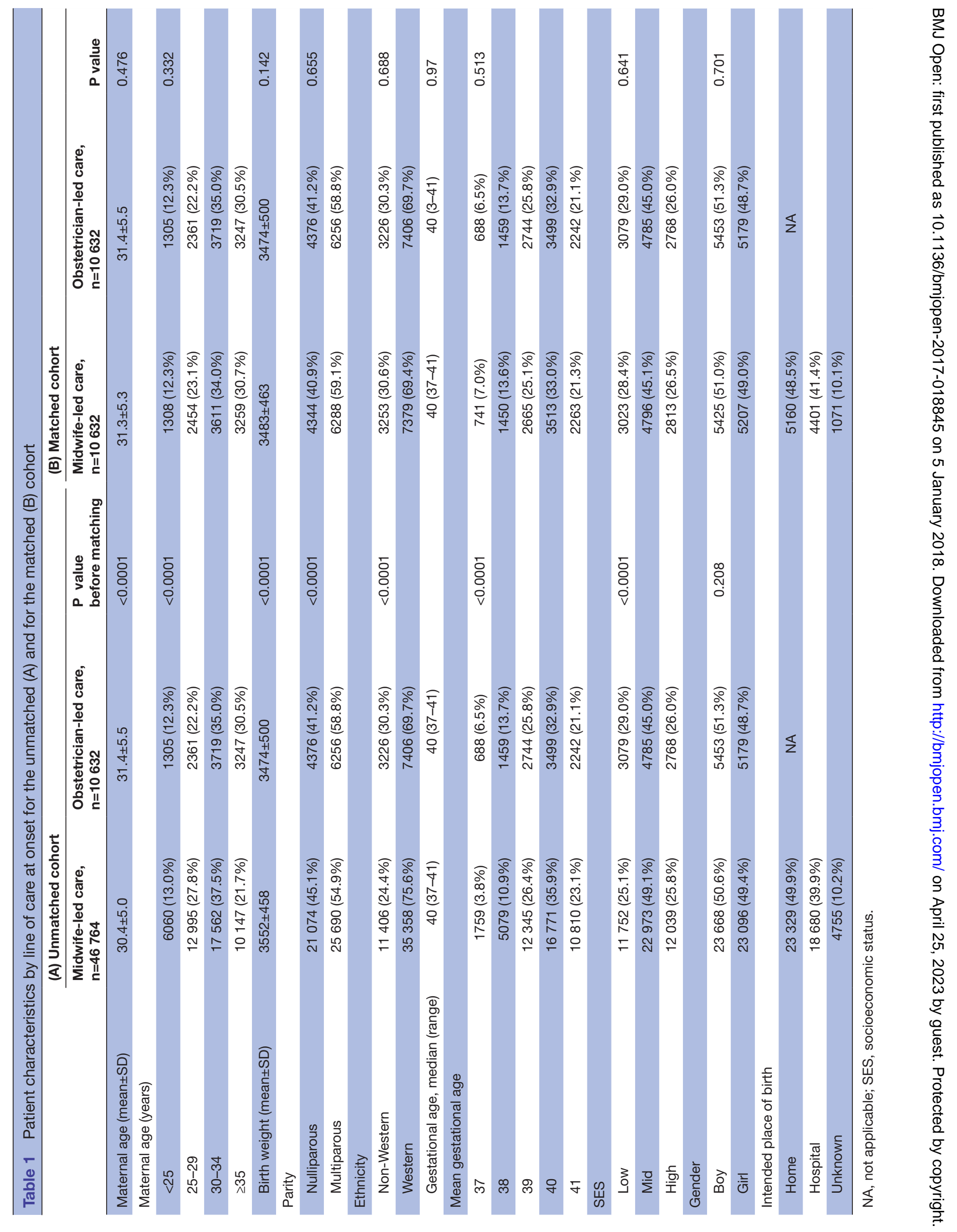


the overlap and region of common support between the two groups. We used significance testing ( $\mathrm{P}$ value) to assess the balance of the covariates after matching.

\section{Baseline and outcome analyses}

Patient characteristics were compared between the groups. Discrete and continuous variables were compared with $\chi^{2}$ tests and Student's t-tests, respectively. For our primary outcome, we calculated the incidence of intrapartum and neonatal mortality up to 28 days of birth, by level of care at onset of labour (midwife led vs obstetrician led). Numerator and denominator were the number of perinatal deaths and the total number of women in each category, respectively. Primary and secondary outcomes for the matched and unmatched cohort were compared. ORs with $95 \%$ (CIs) were generated with the use of logistic regression. When one of the numerators was 0 , that is, when no perinatal death occurred, we added 0.5 to each of the parts of the two by two table.

For the mortality outcome, we performed subgroup analyses by parity (nulliparous vs multiparous) and by intended place of birth (using hospital birth in secondary care as reference). This intended place does not inform us about the actual place of birth.

\section{Clinical overview of perinatal deaths}

We extracted all relevant clinical conditions from each delivery resulting in perinatal death (such as infection and uterine rupture) and described the actual place of death and in case of referral the suspected fetal or neonatal condition on referral, and presented them in an overview table.

\section{Additional analyses}

Information regarding our additional analyses can be found in online supplementary appendix A1. These were analyses based on a random sample to validate our denominator.

All data were analysed using the $\mathrm{R}$ statistical software V.3.2.0 with the Match library for matched analysis and statistical package SAS V.9.3. Two-sided $\mathrm{P}$ values $<0.05$ were considered to indicate statistical significance. In the Dutch system, no ethical approval was needed for this retrospective study. Permission to access the data was granted by the Dutch Perined registry (PRN nr 11_03) and by the involved obstetric and paediatric wards, as well as midwife practices. Data were handled anonymously.

\section{RESULTS}

Within our 3-year time period, a total of 57396 women living in the Amsterdam perinatal healthcare region gave birth at term of a singleton child without congenital anomalies, antepartum fetal death or any of our eight predefined risk factors. Labour started in midwife-led care for 46764 $(81 \%)$ low-risk women and in obstetrician-led care for 10632 (19\%) low-risk women. Thirty-three per cent (15603/46 764) of women who started labour in midwife-led care were transferred to obstetrician-led care during labour. For women with an intended home birth, the intrapartum referral rate was $24 \%$ compared with $37 \%$ for women with an intended hospital birth (data not shown).

\section{Unmatched group}

Baseline characteristics of the unmatched study population are shown in table 1A. Before PSM, the two groups were statistically significantly different for all included variables except for gender of the child. Women in the midwife-led care group at start of labour tended to be younger, more often nulliparous, more often of Western ethnicity, less frequently of low socioeconomic status and were more likely to give birth between 41 and 42 weeks (although median gestational age at birth was comparable), with also higher mean birth weight. Half of all women $(50 \%)$ in the midwife-led care group intended a home birth and $40 \%$ intended midwife-led hospital birth (10\% unknown intended place of delivery). Before matching, the mean $(\mathrm{SD})$ propensity score was $0.18(0.05)$ in the midwife group and $0.20(0.07)$ in the obstetrician group $(\mathrm{P}<0.0001)$.

\section{Case selection}

A total of 41 women suffering perinatal death were identified within the cohort of pregnancies without major risk factors in the PRN database (figure 1). After classification of their records, 14 women were excluded because of one or more exclusion criteria $(n=13)$ or double registration $(n=1)$ in the PRN. From two women, we were unable to retrieve the medical records. In five women, we were unable to distinguish antepartum from intrapartum death, and these women were labelled 'unable to classify'.

With our broader PRN database search to account for possible classification problems and definition differences (Materials and methods section; $n=233$ ), we found eight additional eligible patients for inclusion, and another nine patients were added to the 'unable to classify' group. Data we received from the 18 hospitals in our region led to inclusion of four additional women suffering perinatal death that were not registered in the PRN.

A total of 32 intrapartum and neonatal deaths remained for analysis. The 14 deaths in the 'unable to classify' group were later included in a sensitivity analysis.

\section{Outcomes for the unmatched group}

Of the 32 women who suffered intrapartum and neonatal death $(0.56 \%$ \% $), 30$ had started labour in midwife-led care versus 2 in obstetrician-led care (midwife-led care $0.64 \%$ o (30/46 764), obstetrician-led care $0.19 \%$ (2/10 632) OR 3.4 (95\% CI 0.82 to 14) (table 2A). Subgroup analyses and secondary outcomes are reported in tables $2 \mathrm{~A}$ and $3 \mathrm{~A}$, respectively.

\section{PROPENSITY SCORE ANALYSIS}

For the 10632 women in obstetrician-led care, a total of 10632 women in the midwife-led care group were selected 


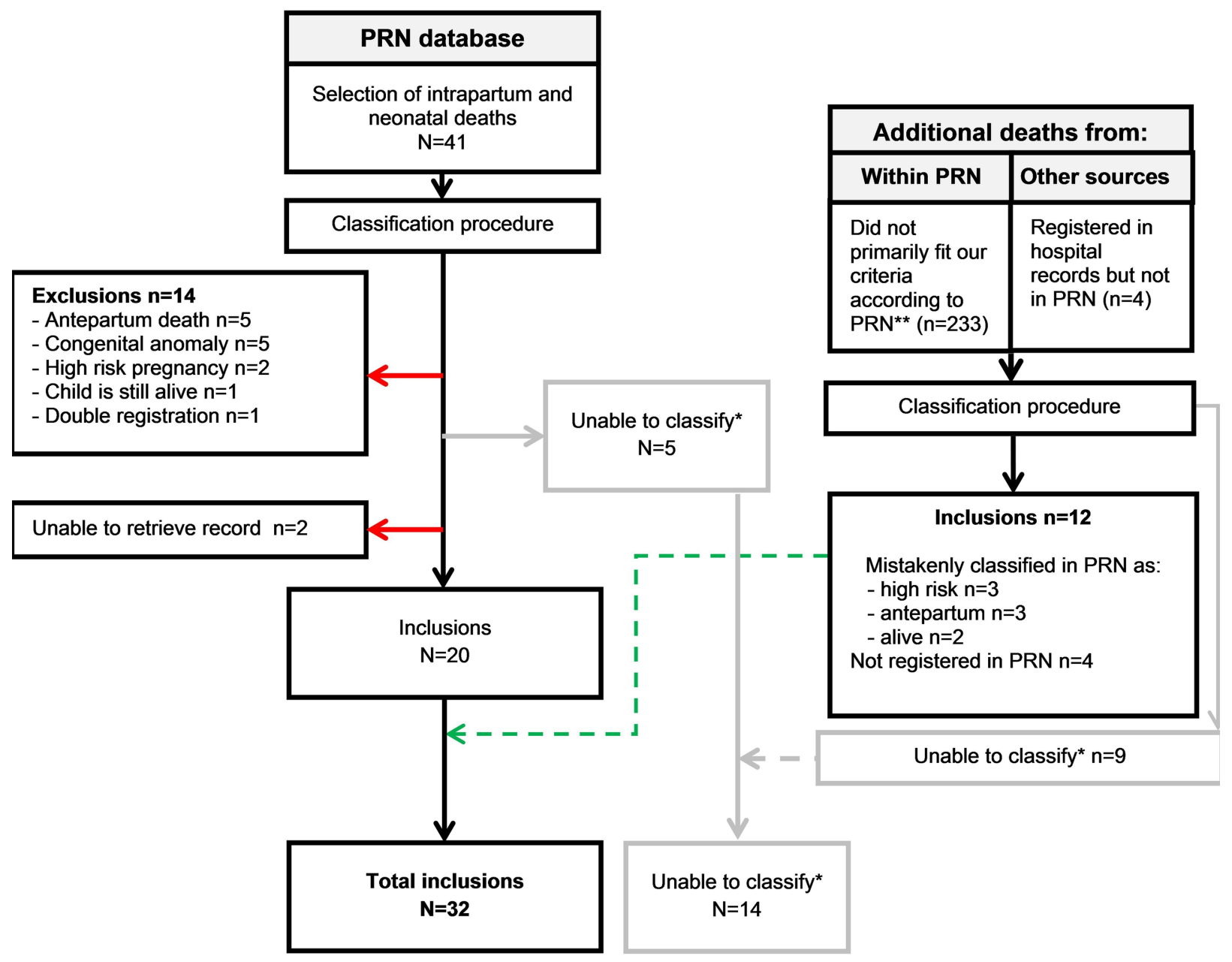

* The classification team was unable to distinguish antepartum and intrapartum death

** cases classified in the PRN as high risk, antenatal stillbirth, congenital anomalies or multiple pregnancy, although not subject of this study, were verified for eligibility to double-check the classification from the PRN.

Figure 1 Flow chart selection of perinatal deaths. PRN, perinatal register.

as control (1:1 ratio). Propensity scores were generated by using the following patient variables: maternal age, birth weight, parity, ethnicity, gestational age, socioeconomic status and gender of the child. Propensity score distribution was plotted to verify overlap and the region of common support between the two groups (online supplementary appendix B figure 1). After matching, the mean propensity score was $0.20(0.06)$ in the midwife group and $0.20(0.06)$ in the obstetric care group $(\mathrm{P}=0.37)$. The baseline maternal characteristics after PSM are shown in table 1B and were comparable between the midwife-led and obstetrician-led groups.

A repeat of our perinatal mortality analyses for the PSM cohort did not significantly alter our primary and subgroup-analysis findings (midwife-led care $0.75 \%$ o (8/10 632); obstetrician-led care $0.19 \%$ (2/10 632), OR $4.0,95 \%$ CI 0.85 to 18.9 ; table $2 \mathrm{~B}$ ).

Subgroup analysis for parity showed an OR of 2.8 (95\% CI 0.72 to 16.8 ) for perinatal mortality in the nulliparous group and an OR of 5.0 (95\% CI 0.24 to 104) for the multiparous group.

Subgroup analyses by intended place of birth showed an OR of 3.1 (95\% CI 0.52 to 18.5$)$ of perinatal mortality for the subgroup of women with an intended home birth in the midwife-led care group, when compared with the women in the obstetrician-led care group (table 2B). For women with an intended hospital birth, the OR was 4.8 (95\% CI 0.89 to 26.4). For one women with perinatal death, the intended place of birth was unknown.

Table 3B shows the secondary outcomes after propensity score matching. The risk of NICU admittance was reduced in the midwife-led care group $(0.2 \%$ vs $0.4 \%$; OR $0.44 ; 95 \%$ CI 0.26 to 0.75 ) with no statistically significant difference in 5 min Apgar scores $<7 \quad(0.70 \%$ vs $0.88 \%$; table 3B). Intervention rates (including CS, instrumental birth, epidural analgesia, manual placenta removal and episiotomy) were reduced in the midwife-led care group, as was the risk of postpartum haemorrhage. 


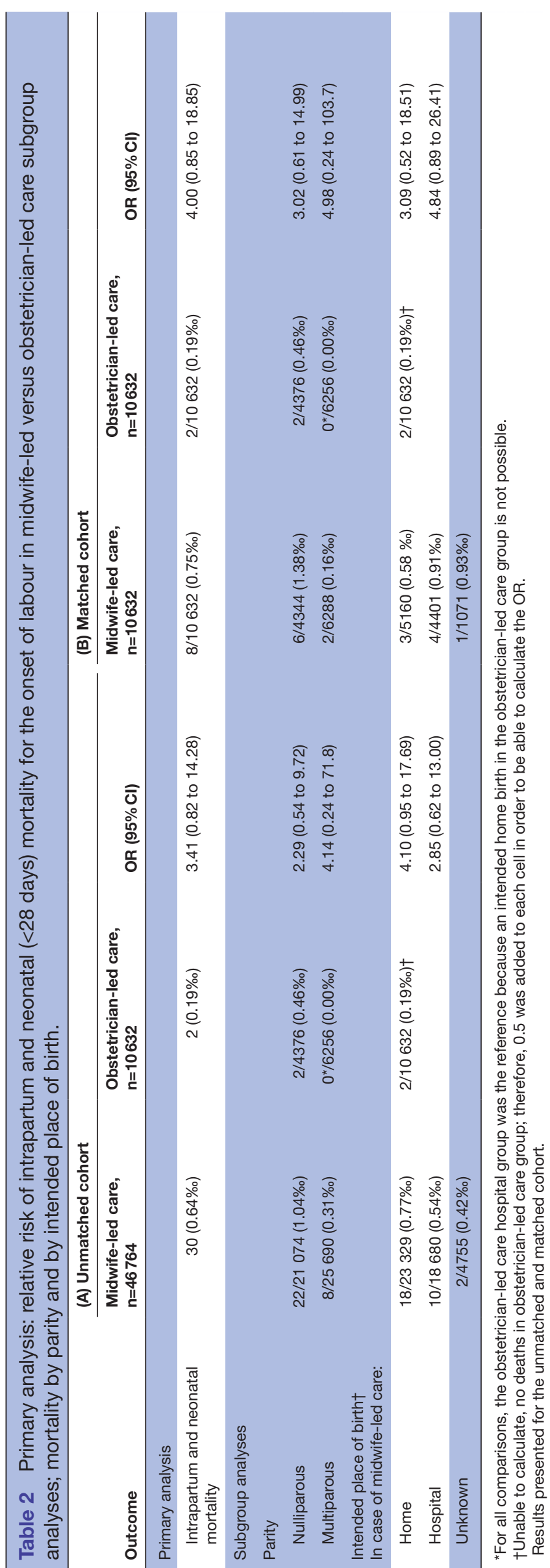

\section{Clinical overview of perinatal deaths}

There were nine (28\%) intrapartum deaths, while 23 babies $(72 \%)$ were born alive but died within 7 days $(n=20)$ or between 7 and 27 days $(n=3)$. Seven children $(22 \%)$ were born after CS, and six (19\%) after instrumental delivery. Nine neonates (28\%) were admitted to an NICU for at least 24 hours. An overview of the most relevant clinical diagnoses of the included perinatal deaths can be found in online supplementary appendix C. Asphyxia without a further known or specified cause was the most commonly reported clinical condition $(n=17)$ followed by infection $(n=6)$. There were two neonatal deaths in the obstetrician-led care group. For both women, the medical indication was a request for sedation for prelabour uterine contractility. One neonate died of asphyxia, and the other of a combination of asphyxia and group B streptococcal (GBS) sepsis.

\section{Additional analyses}

None of the additional validation or sensitivity analyses significantly altered our results (online supplementary appendix tables D1-D3).

\section{DISCUSSION}

In this retrospective cohort study among low-risk women, perinatal mortality occurred in $0.64 \%$ of the women in midwife-led care and $0.19 \%$ of the women in obstetrician-led care (OR 3.4, 95\% CI 0.82 to 14.3). A PSM analysis, as well as subgroup analysis for parity or intended place of birth did not alter this result, with a consistent (but statistically non-significant) higher mortality in the midwife-led group.

\section{Strengths and limitations}

An important strength of our study is that we thoroughly checked for the completeness of our data with additional sources of the medical records. We performed extensive validity checks to ensure that our denominators were reliable. Other strengths are the multidisciplinary approach, and the fact that we created comparable groups through propensity score matching, thereby attempting to reduce bias due to confounding variables such as age, parity, socioeconomic status and ethnicity.

Our study relevantly exposed the shortcomings of using aggregated data from our national perinatal database (PRN) ${ }^{16}$ It is unclear whether the seriousness of an adverse outcome influences the reliability of the registration. Furthermore, certain important risk factors for perinatal mortality, such as level of education, smoking during pregnancy and maternal body mass index, were not available from the registry and therefore not included in this study.

Defining the 'low-risk' population in our obstetrician-led care group was challenging. Women were considered low risk if they had no major risk factors, but this was not always reliably registered in our national database. With PSA, we further aimed to reduce imbalance 


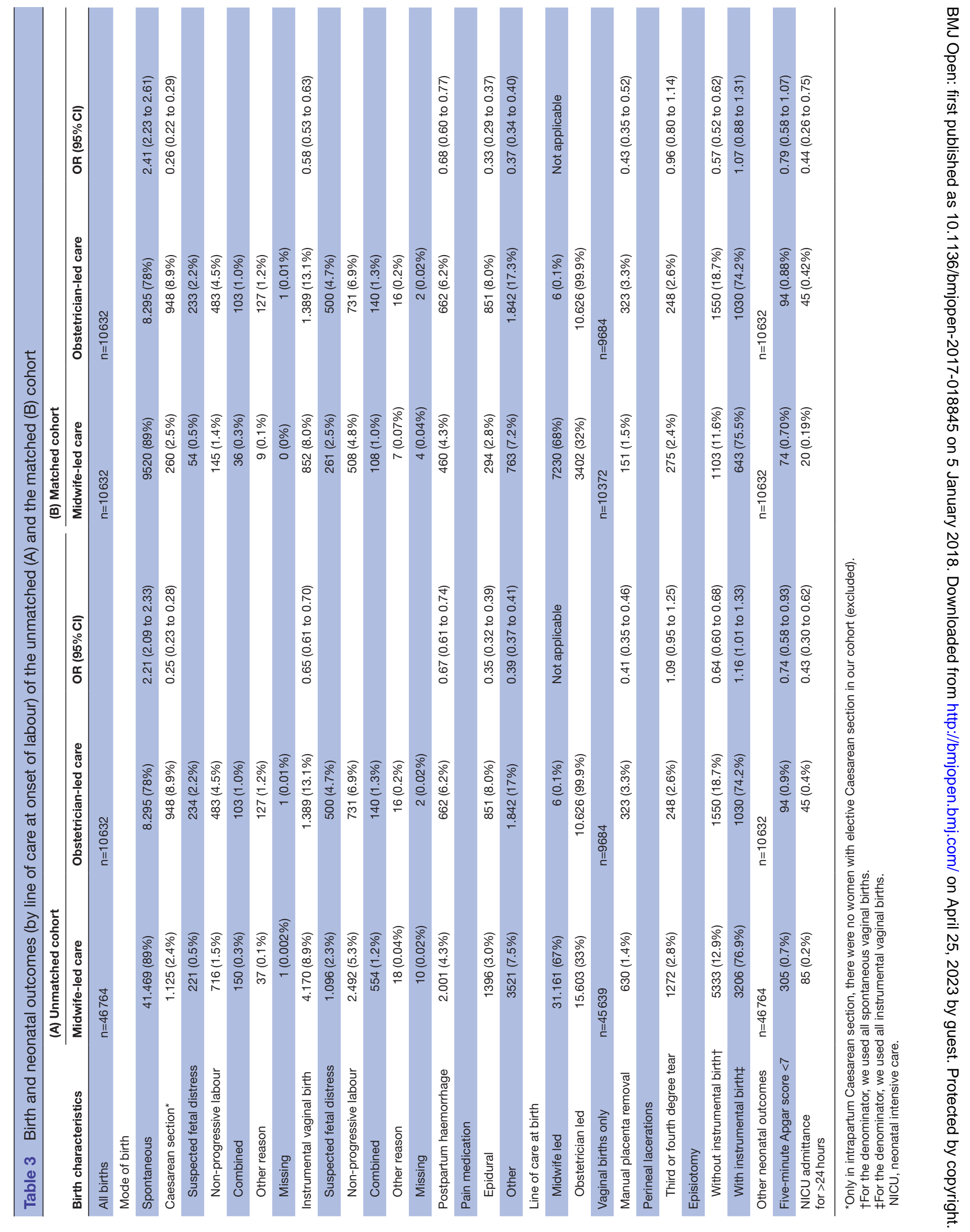


in baseline characteristics between the groups. Results of the PSA were similar to the unadjusted analysis, to a conventional multivariate logistic regression analysis and to conditional regression analysis (data not shown).

Although our denominators were substantial, the large CIs in our primary mortality analyses overlapping 1 indicate limited power. A post hoc analysis showed that our study had the power to exclude a difference in mortality of 0.8 per 1000 between groups, based on a non-inferiority design with an alpha of 0.025 , beta of 0.2 and a mortality incidence of 0.6 per 1000 . As a consequence, the mortality difference of 0.45 per 1000 that we observed was not statistically significant. It is important to stress that Dutch women can make a choice between midwife-led care and obstetrician-led care, with midwifery-led care as the default for low-risk women. As such, the level of care has a safety dimension, and a significance level of 0.05 is not a prerequisite for safety. ${ }^{1718}$ For this study, we focused on mortality after the onset of labour, meaning that we are unable to draw any conclusions regarding antepartum fetal death and total perinatal mortality. It would be very interesting to review this in a separate study, as antepartum death is the largest group. Our data referred to the period 1 January 2005 up to 31 December 2007. Data from 2008 onwards were not yet available in the national database when we started the project. Perinatal mortality rates in the Netherlands, both preterm and at term, have decreased over time. This was observed for fetal death and for neonatal death, with a steady decline of the term perinatal mortality risk (including prepartum) of 3.8\%o in 2001 to $1.9 \%$ in $2014 .{ }^{19}$ Relevant changes within the Dutch obstetric system during and after our study period include the introduction of routine structural 20 weeks anomaly scan (January 2007), therapeutic neonatal hypothermia for perinatal asphyxia (2009), the installation of the Foundation Perinatal Audit in the Netherlands with a multidisciplinary approach to critically and systematically audit term perinatal deaths (2010) and the installation of the College Perinatal Care to implement the recommendations of the steering group 'A good start' with the aim to reduce maternal and perinatal mortality (2011). Although the decline in perinatal mortality is a substantial improvement, the 2010 ranking relative to other European countries showed only modest progression and need for further for improvement. ${ }^{20}{ }^{21}$ Since we studied a specific (matched) population of low-risk women in a region of the Netherlands, generalisability of our findings to a broader population needs further investigation.

\section{Interpretation}

Our findings are partially in line with other studies in this field, although study designs differ. The Dutch study of Evers et al performed in a similar setting reported an increased intrapartum and early neonatal mortality risk in the midwife-led low-risk group of (26/18 686) $1.4 \%$, as compared with (10/16 739) $0.60 \%$ in obstetrician-led care, while the latter group contained a mixed low-risk and high-risk group. A limitation of that study was that the numerator and the denominator were not taken from exactly the same geographical region. ${ }^{22}$ Also, Evers et al did not perform an analysis in which the obstetrician-led population was limited to low-risk women. However, the 10 women in their study that suffered an intrapartum or early neonatal death after start of labour in obstetrician-led care all had risk factors, implicating that the low-risk women in obstetrician-led care did not suffer perinatal mortality (A Kwee, personal communication, 2017). ${ }^{8}$ As the women in midwife-led care in Evers' study obviously were low risk, the difference in perinatal mortality risk between midwife-led and obstetrician-led care was $1.4 \%$, which is twice as high as our study.

The prospective Birthplace in England study group analysed a composite poor neonatal outcome (including perinatal mortality) among low-risk women and found no difference in incidence between obstetric units $(81 / 19551,4.4 \% 0)$ and other birthplaces, including homebirth (70/16 553, 4.2\%o; adjusted OR 1.16, 95\% CI 0.76 to 1.77$).{ }^{6}$ The composite outcome however did occur more in nulliparous women having homebirths. A population-based retrospective cohort study in the USA by Snowden et al found that planned out-of-hospital birth was associated with a higher rate of perinatal death than planned inhospital birth (3.9\%o vs $1.8 \%$, adjusted OR $2.43 ; 95 \%$ CI 1.37 to 4.30$){ }^{23}$

Based on the direction of the OR towards increased perinatal mortality in the midwife-led care group in our study, it would be expected that morbidity outcomes would point in the same direction. Instead, we found fewer NICU admissions and less babies with low Apgar scores in midwife-led care. Both are surrogate outcomes for neonatal morbidity with a subjective component that might specifically play a role when comparing different levels of care. ${ }^{24}{ }^{25}$ In the absence of specific objective morbidity outcomes, we are unable to draw any conclusions regarding neonatal morbidity among midwife-led deliveries. Our study found instrumental delivery rates to be significantly higher in the obstetrician-led care group, which is in line with other reports. ${ }^{6}{ }^{23}$ Previous studies show that intervention rates within midwife-led care also differ based on intended place of birth, with less interventions in the intended homebirth group. ${ }^{57}$ A next step should be an analysis of the reasons for the difference. We hypothesise that continuous presence of an obstetric care provider from the onset of labour and continuous CTG monitoring are important determinants of the differences, both with respect to perinatal mortality as well as with the number of instrumental deliveries. It is plausible that the increase in interventions in the obstetrician-led care are directly related to the (not statistically significant) decreased mortality rates.

Perinatal asphyxia and infection were the two most common relevant conditions of all of the included perinatal deaths, consistent with existing literature ${ }^{26}$ Prevention, early detection and early intervention in situations leading to asphyxia and infection during labour have the potential of further reducing perinatal mortality at term. 
While our findings were not statistically significant and do not allow for conclusions regarding causality, the results are concerning and warrant further evaluation of the underlying causes and replication.

\section{Author affiliations}

${ }^{1}$ Department of Obstetrics and Gynaecology, Academic Medical Center, Amsterdam, The Netherlands

${ }^{2}$ Department of Obstetrics and Gynaecology, Leiden University Medical Center, Leiden, The Netherlands

${ }^{3}$ Department of Medical Informatics, Academic Medical Center, Amsterdam, The Netherlands

${ }^{4}$ Department of Obstetrics and Gynaecology, VU University Medical Center, Amsterdam, The Netherlands

${ }^{5}$ Neonatal Intensive Care, Emma Children's Hospital AMC, Amsterdam, The Netherlands

${ }^{6}$ School of Paediatrics and Reproductive Health, The Robinson Institute, University of Adelaide, Adelaide, South Australia, Australia

${ }^{7}$ South Australian Health and Medical Research Institute, Adelaide, South Australia, Australia

Acknowledgements We thank all midwives, gynaecologists, neonatologists and members of the PRN registry for collecting data on perinatal mortality in the region of Amsterdam. We also thank Dr M G A J Wouters, perinatologist, for his help with classification of the patient records.

Contributors All authors (MMJW, BYvdG, ACJR, FCDB, JAMvdP, PT and BWM) contributed to the realisation of the protocol. ACJR and MW collected all data from the PRN database. MW and BYvdG retrieved additional data from hospitals and midwife practices. BYvdG, FCDB, MMJW, JAMvdP and BWM formed teams of professionals together with three midwives in primary care and an additional perinatologist, and selected cases for inclusion. They also reviewed all 100 cases for the validity check. Data analysis was done by MMJW and ACJR. Paper was written by MMJW in close collaboration with BWM, JAMvdP, ACJR and BYvdG. All authors approved the final manuscript.

Funding BWM is supported by a NHMRC Practitioner Fellowship (GNT1082548).

Competing interests None declared.

Provenance and peer review Not commissioned; externally peer reviewed.

Data sharing statement № additional data are available.

Open Access This is an Open Access article distributed in accordance with the Creative Commons Attribution Non Commercial (CC BY-NC 4.0) license, which permits others to distribute, remix, adapt, build upon this work non-commercially, and license their derivative works on different terms, provided the original work is properly cited and the use is non-commercial. See: http://creativecommons.org/ licenses/by-nc/4.0/

(c) Article author(s) (or their employer(s) unless otherwise stated in the text of the article) 2018. All rights reserved. No commercial use is permitted unless otherwise expressly granted.

\section{REFERENCES}

1. CVZ. Verloskundig vademecum 2003. Eindrapport van de commissie verloskunde van het college voor zorgverzekeringen. [Obstetrical manual 2003. Final report of the obstetric working group of the national health insurance board CVZ]. Diemen: CVZ, 2003.

2. Registry TNP. Perinatal care in the Netherlands 2013. Utrecht: Stichting perinatale registratie Nederland, 2013.

3. de Jonge A, van der Goes BY, Ravelli AC, et al. Perinatal mortality and morbidity in a nationwide cohort of 529,688 low-risk planned home and hospital births. BJOG 2009;116:1177-84.
4. van der Kooy J, Poeran J, de Graaf JP, et al. Planned home compared with planned hospital births in the Netherlands: intrapartum and early neonatal death in low-risk pregnancies. Obstet Gynecol 2011;118:1037-46.

5. Janssen PA, Saxell L, Page LA, et al. Outcomes of planned home birth with registered midwife versus planned hospital birth with midwife or physician. CMAJ 2009;181:377-83.

6. Brocklehurst P, Hardy P, Hollowell J, et al. Perinatal and maternal outcomes by planned place of birth for healthy women with low risk pregnancies: the Birthplace in England national prospective cohort study. BMJ 2011;343:d7400.

7. Hutton EK, Cappelletti A, Reitsma AH, et al. Outcomes associated with planned place of birth among women with low-risk pregnancies. CMAJ 2016;188:E80-E90.

8. Evers AC, Brouwers HA, Hukkelhoven CW, et al. Perinatal mortality and severe morbidity in low and high risk term pregnancies in the Netherlands: prospective cohort study. BMJ 2010;341:c5639.

9. Wiegerinck MM, van der Goes BY, Ravelli AC, et al. Intrapartum and neonatal mortality in primary midwife-led and secondary obstetrician-led care in the Amsterdam region of the Netherlands: A retrospective cohort study. Midwifery 2015;31:1168-76.

10. Méray N, Reitsma JB, Ravelli AC, et al. Probabilistic record linkage is a valid and transparent tool to combine databases without a patient identification number. J Clin Epidemiol 2007;60:883.e1-883. e11.

11. Registry TNP. Perinatal care in the Netherlands 2007. Utrecht: Stichting perinatale registratie Nederland, 2009.

12. Eskes M, Waelput AJ, Erwich JJ, et al. Term perinatal mortality audit in the Netherlands 2010-2012: a population-based cohort study. BMJ Open 2014;4:e005652.

13. ROSENBAUM PR, RUBIN DB. The central role of the propensity score in observational studies for causal effects. Biometrika 1983;70:41-55.

14. Garrido MM, Kelley AS, Paris J, et al. Methods for constructing and assessing propensity scores. Health Serv Res 2014;49:1701-20.

15. Gilbert SA, Grobman WA, Landon MB, et al. Elective repeat cesarean delivery compared with spontaneous trial of labor after a prior cesarean delivery: a propensity score analysis. Am J Obstet Gynecol 2012;206:311.e1-311.e9.

16. de Jonge $\mathrm{A}$, Wouters $\mathrm{M}$, Klinkert $\mathrm{J}$, et al. Pitfalls in the use of registerbased data for comparing adverse maternal and perinatal outcomes in different birth settings. BJOG 2017;124:1477-80.

17. Buyse M, Hurvitz SA, Andre F, et al. Statistical controversies in clinical research: statistical significance-too much of a good thing. Ann Oncol 2016;27:760-2.

18. Nuzzo R. Scientific method: statistical errors. Nature 2014;506:150-2.

19. Perined. Perinatal audit of term mortality in 2013 \& 2014, 2016. [Perinatale audit van à terme sterfte in 2013 \& 2014].

20. Mohangoo AD, Buitendijk SE, Hukkelhoven CW, et al. [Higher perinatal mortality in The Netherlands than in other European countries: the Peristat-II study]. Ned Tijdschr Geneeskd 2008;152:2718-27.

21. Ravelli AC, Tromp M, van Huis M, et al. Decreasing perinatal mortality in The Netherlands, 2000-2006: a record linkage study. J Epidemiol Community Health 2009;63:761-5.

22. Jonge A, Mol BW, van der Goes BY, et al. Too early to question effectiveness of Dutch system. BMJ 2010;341:c7020.

23. Snowden JM, Tilden EL, Snyder J, et al. Planned out-of-hospital birth and birth outcomes. N Engl J Med 2015;373:2642-53.

24. Wiegerinck MM, Danhof NA, Van Kaam AH, et al. The validity of the variable 'NICU admission' as an outcome measure for neonatal morbidity: a retrospective study. Acta Obstet Gynecol Scand 2014;93:603-9.

25. Grünebaum A, McCullough LB, Brent RL, et al. Justified skepticism about Apgar scoring in out-of-hospital birth settings. J Perinat Med 2015;43:455-60.

26. Grünebaum A, McCullough LB, Arabin B, et al. Underlying causes of neonatal deaths in term singleton pregnancies: home births versus hospital births in the United States. J Perinat Med 2017;45:349-57. 